\title{
Orthologs of the small RPB8 subunit of the eukaryotic RNA polymerases are conserved in hyperthermophilic Crenarchaeota and "Korarchaeota"
}

\author{
Eugene V Koonin*1, Kira S Makarova ${ }^{1}$ and James G Elkins ${ }^{2}$
}

Address: ${ }^{1}$ National Center for Biotechnology Information, National Library of Medicine, National Institutes of Health, Bethesda, MD 20894, USA and ${ }^{2}$ Microbial Ecology and Physiology Group, Biosciences Division, Oak Ridge National Laboratory, Oak Ridge, TN 37831, USA

Email: Eugene V Koonin* - koonin@ncbi.nlm.nih.gov; Kira S Makarova - makarova@ncbi.nlm.nih.gov; James G Elkins - elkinsjg@ornl.gov

* Corresponding author

Published: 14 December 2007

Biology Direct 2007, 2:38 doi:10.1186/1745-6150-2-38

This article is available from: http://www.biology-direct.com/content/2/1/38

(c) 2007 Koonin et al; licensee BioMed Central Ltd.

This is an Open Access article distributed under the terms of the Creative Commons Attribution License (http://creativecommons.org/licenses/by/2.0), which permits unrestricted use, distribution, and reproduction in any medium, provided the original work is properly cited.
Received: 13 December 2007

Accepted: I4 December 2007

\begin{abstract}
: Although most of the key components of the transcription apparatus, and in particular, RNA polymerase (RNAP) subunits, are conserved between archaea and eukaryotes, no archaeal homologs of the small RPB8 subunit of eukaryotic RNAP have been detected. We report that orthologs of RPB8 are encoded in all sequenced genomes of hyperthermophilic Crenarchaeota and a recently sequenced "korarchaeal" genome, but not in Euryarchaeota or the mesophilic crenarchaeon Cenarchaeum symbiosum. These findings suggest that all 12 core subunits of eukaryotic RNAPs were already present in the last common ancestor of the extant archaea.
\end{abstract}

Open peer review: This article was reviewed by Purificacion Lopez-Garcia and Chris Ponting.

\section{Findings}

The core components of the information-processing systems, and in particular, the transcription machinery, are conserved between archaea and eukaryotes, and distinct from the bacterial versions. The heteromultimeric eukaryotic RNAPs consist of 12 subunits (Rpb1-12), of which 11 are conserved in archaea and eukaryotes whereas one, $\mathrm{Rpb} 8$, is thought to be unique for eukaryotes [1-6]. Rpb8 is a small protein that typically consists of $\sim 120-150$ amino acids and shows relatively poor sequence conservation in eukaryotes. The structure of Rpb8 has been solved, originally, by solution NMR [7] and, subsequently, as part of the RNAP II core, by X ray crystallography [8]. The two structures are in good agreement and indicate that Rpb8 forms a distinct version of the $\mathrm{OB}$ (oligonucleotide-oligosaccharide-binding) fold [9] that is characterized by a distinct pattern of $9 \beta$-strands and a pair of invariant glycines in the turn between strands 7 and 8 . In the RNAP II structure, Rpb8 interacts with the so-called pore module of Rpb1 at a defined motif that is conserved in both eukaryotic and archaeal Rpb1 orthologs [8]. In addition, Rpb8 genetically interacts with another small subunit, Rpb6, that is also adjacent to the pore module in the core RNAP structure [6]. It has been suggested that Rpb8 and Rpb6, together with the pore module, form a distinct functional unit [6]. The exact role of the small subunits remains unknown although both Rpb6 and Rpb8 are conserved in all eukaryotes, are shared between RNAP I, II and III, and are essential for yeast growth. Regardless of the precise function of Rpb8, the purported absence of this RNAP subunit ortholog in archaea is the major gap in the picture of the otherwise exact correspondence between the core transcriptional machineries of eukaryotes and archaea, and is highly unexpected considering the conservation of all other subunits including the functional partner of Rpb8, Rpb6. 
In the course of the genome annotation for the first sequenced member of the "Korarchaeota", a putative deep branch of archaea ([10] and manuscript in preparation), one of us (JGE) identified a short (110 amino acids) predicted protein for which some of the best hits in a BLAST search [11] were the eukaryotic Rpb8 subunits. Although the sequence similarity was not statistically significant, this observation prompted a systematic search for possible archaeal homologs of Rpb8. BLAST searches started with the sequences of Rpb8 subunits of various eukaryotic species, again, showed statistically not significant similarity to a distinct set of crenarchaeal proteins that were similar in size to (or slightly shorter than) Rpb8. However, reciprocal iterative PSI-BLAST searches (inclusion E-value cut-off 0.01) started with some of these crenarcheal sequences showed significant similarity to Rpb8. For example, a search with the sequence from Hyperthermus butylicus (Hbut_0467) used as the query retrieved the Rpb8 sequence from fission yeast Schizosaccharomyces pombe in the $2^{\text {nd }}$ iteration, with a E-value of 0.004 , and numerous eukaryotic Rpb8 sequences in the $3^{\text {rd }}$ iteration, with highly significant E-values. Similarly, a search with the sequence from Igniococcus hospitalis (Igni_1165) retrieved Rpb8 from Entamoeba histolytica in the $5^{\text {th }}$ iteration, with an E-value of $4 \times 10^{-5}$ ).

Examination of a multiple alignment of the Rpb8 sequences from diverse eukaryotes and the putative archaeal counterparts showed remarkable conservation of all elements of the OB-fold, in particular, the diagnostic motif between strands 7 and 8 (although one of the two glycines that are invariant in Rpb8 is replaced in a subset of the putative archaeal homologs (Fig. 1a). Furthermore, secondary structure prediction for the archaeal proteins showed a near-perfect superposition with the secondary structure elements extracted from the crystal structure of Rpb8 [8] (Fig. 1a). Taken together, these findings indicate that the detected small archaeal proteins are bona fide homologs of Rpb8.

Small proteins homologous to Rpb8 were identified in all 10 sequenced genomes of hyperthermophilic Crenarchaeota and the only available korarchaeal genome. Each of these genomes encodes a single Rpb8 homolog which mimics the situation in eukaryotes where no paralogs of Rpb8 are detectable. By contrast, $n$ homologs of these proteins were identified in Euryarchaeota and the mesophilic crenarchaeon Cenarchaeum symbiosum, despite extensive search including running a position-specific scoring matrix for Rpb8 and their crenarchaeal-korachaeal homologs against a dedicated database of euryarchaeal and C. symbiosum protein sequences. Thus, we conclude that all thermophilic Crenarchaeota and at least one korarchaeote encode a single ortholog of eukaryotic Rpb8 whereas Euryarchaeota and C. symbiosum (the only mes- ophilic crenarchaeon for which the genome sequence is currently available) do not.

In retrospect, we became aware that the protein we identified as the crenarchaeal ortholog of Rpb8 has already been described as one of the 13 experimentally defined subunits of the RNAP of the crenarchaeon Sulfolobus acidocaldarius and designated RpoG [12], and the ortholog encoded in the genome of $S$. solfataricus has been accordingly annotated [13]. Given these data, it appears sensible to adopt the designation RpoG for the archaeal orthologs of Rpb8. In a subsequent global analysis of mRNA stability in the two Sulfolobus species, it has been shown that the RpoG mRNA is markedly more stable than mRNAs of other RNAP subunits, and the apparent uniqueness of this subunit in Sulfolobus has been emphasized [14]. The present analysis clarifies the situation by showing that RpoG is conserved throughout the hyperthermophilic Crenarchaeota (and at least one korarchaeote).

Although the small size of Rpb8 and its archaeal orthologs (RpoG) hampers reliable phylogenetic analysis, the maximum likelihood tree we constructed shows a clear separation of the eukaryotic and crenarchaeal branches, and within the latter, the split between Thermoproteales and Sulfolobales (Fig. 1b). Interestingly, the korarchaeal RpoG clustered with Thermoproteales in a strongly supported branch (Fig. 1b). Broader implications of this observation for the evolution of the "Korarchaeota" remain to be investigated.

In the archaeal genomes, the $r p o G$ gene is embedded in a notable, partially conserved genomic context (Fig. 1b). With few exceptions, $r p o G$ forms either a codirectional or a divergent but potentially coregulated gene pair with a gene encoding a small RNA-binding protein (COG1958) that is orthologous to eukaryotic Lsm6 and is implicated in RNA-processing. In most of the Sulfolobales, the latter gene is adjacent to a gene for a tRNA modification enzyme, queuine/archaeosine tRNA-ribosyltransferase. Another common neighbor of $r p o G$ is the gene for transcription elongation factor TFIIIB, with a divergent orientation in the majority of Thermoproteales and a codirectional orientation in the korarchaeote. Finally, Thermofilum pendens appears to have a more complex operon organization, with the genes for another RNAP subunit, a transcription factor and a ribosomal protein in the same predicted operon with rpoG (Fig. 1b). This genomic context suggests that, in Crenarchaeota, RpoG is likely to be involved in a tight functional cooperation with TFIIIB, and could also contribute to coupling transcription with RNA processing and modification.

The finding described here fills the last gap in the one-toone correspondence between the RNAP subunits of 

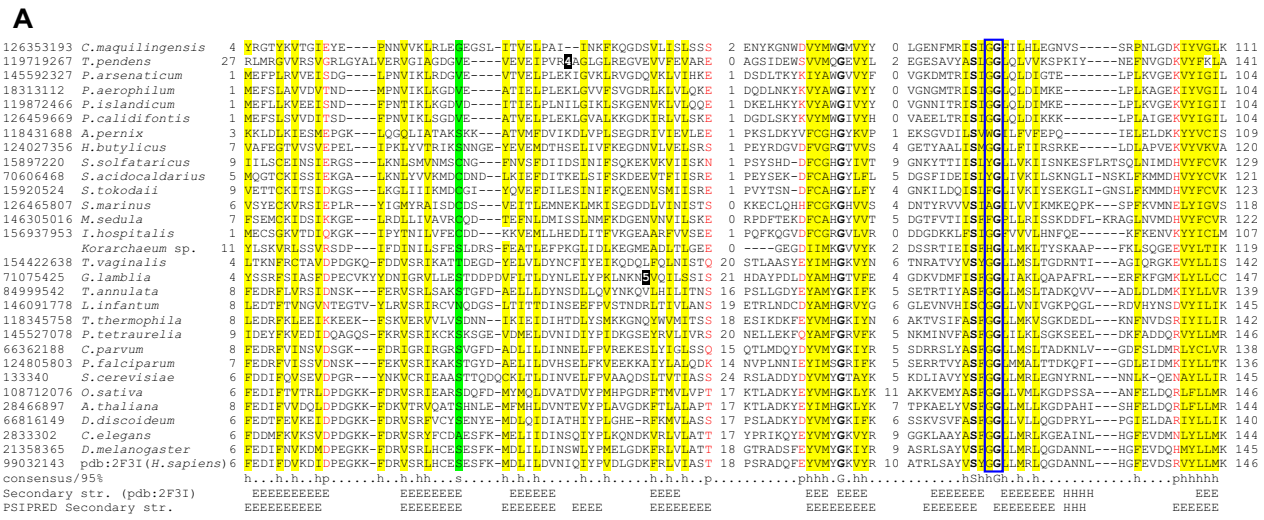

B

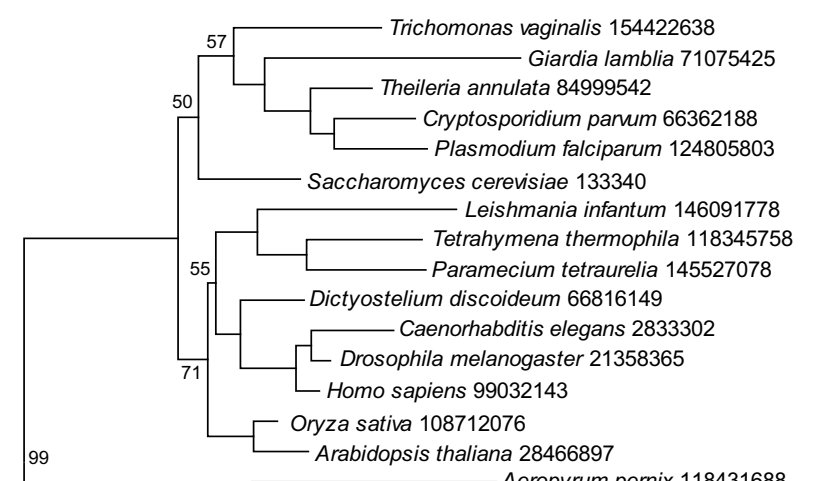

$99 \quad$ Arabidopsis thaliana 28466897
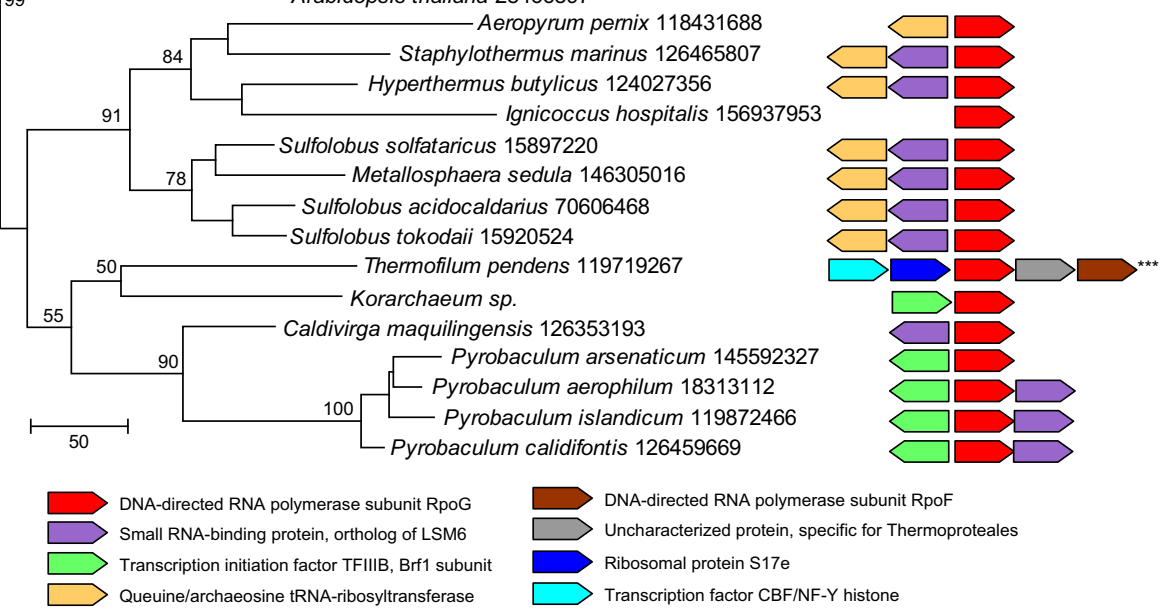

\section{Figure I}

Orthologs of Rpb8 in archaea. (a) Multiple alignment of eukaryotic Rpb8 subunits and their archaeal orthologs (RpoG). The alignment was constructed using the combination of the results obtained with PROMALS [15] and MUSCLE [16], followed by manual correction on the basis of secondary structure prediction that was obtained using PSIPRED [I7] and local alignments generated by PSI-BLAST. Sequences are denoted by their numeric Genbank Identifiers (GI numbers) and species names. The full species names are given in Figure 2. The positions of the first and the last residues of the aligned region in the corresponding protein are indicated for each sequence. The numbers within the alignment represent poorly conserved inserts that are not shown. The numbers of omitted amino acids for $T$. pendens and $G$. lamblia are indicated by reverse shading. Positions with identical amino acids in all aligned sequences are in bold face. The coloring is based on the consensus shown underneath the alignment; ' $h$ ' indicates hydrophobic residues (ACFILMVWYH), ' $p$ ' indicates polar residues (STEDKRNQH), 's' indicates small residues (AGCVDS). Secondary structure is shown for the crystal structure of human Rpb8 (pdb 2F3I); ' $H$ ' indicates $\alpha$-helix and 'E' indicates extended conformation ( $\beta$-strand). The PSIPRED secondary structure prediction is shown underneath the experimental secondary structure. The glycine doublet that is invariant in eukaryotic Rpb8 sequences is boxed. (b) Phylogenetic and genomic contexts of Rpb8/RpoG. The maximum likelihood phylogenetic tree of Rpb8/RpoG was constructed by local rearrangement of an original minimum evolution (Fitch) tree [18] using the MOLPHY program [19]. MOLPHY was also used to compute RELL bootstrap probabilities, which are indicated (as percentages) for selected major branches. Each terminal node of the tree is labeled by the full species name and the GI number. The genomic neighborhoods of the rpoG gene in Crenarchaeota and the "korarchaeal" genome are shown to the right of the respective branches of the tree. Orthologous genes are shown by arrows of the same color. 
archaea and eukaryotes, with the implication that the archaeal "parent" of eukaryotes already possessed the intricate 12-subunit organization of RNAP. Surprisingly, however, Euryarchaeota and the only available genome of a mesophilic crenarchaeon appear to lack an ortholog of Rpb8, a conclusion that is compatible with the report on the reconstruction of a fully active RNAP of the euryarchaeon Methanocaldococcus jannaschii from 12 recombinant proteins which, obviously, did not include Rpb8 [1]. Depending on the adopted evolutionary scenario, it is conceivable that Rpb8 emerged in the crenarchaeal lineage or, perhaps, more plausibly, that it was already present in the common ancestor of all extant archaea but lost at the base of the euryarchaeal branch. Regardless of the solution to this conundrum, experimental study of functional differences between RNAPs of Euryarchaeota and Crenarchaeota should be illuminating, given the unusual difference in their predicted subunit composition.

\section{Abbreviations}

RNAP: RNA polymerase.

\section{Competing interests}

The author(s) declare that they have no competing interests.

\section{Reviewers' reports}

Reviewer 1: Purificacion Lopez-Garcia, Universite ParisSud

This manuscript reports the identification of genes orthologous to RPB8 in archaea. This observation is very interesting and worth publishing, as RPB8 was the only protein conserved in the eukaryotic core of RNA polymerases I, II and III for which orthologues in archaea had not been found. The fact that this protein is apparently missing from Euryarchaeota is intriguing, suggesting that either its role can be fulfilled by other elements or that its primary sequence has evolved beyond recognition. At any rate, the finding of archaeal RPB8 homologues indicates that the complete RNA polymerase core in both archaea and eukaryotes share a common ancestry.

\section{Reviewer 2: Chris Ponting, Oxford University}

This manuscript demonstrates convincingly the presence of an orthologue of eukaryotic RPB8 in hyperthermophilic Crenarchaeota. As such, it provides the last missing piece in the RNAP "puzzle" and should thus be of interest to many working in this field.

\section{Authors' contributions}

EVK contributed to sequence analysis and wrote the manuscript; KSm contributed to sequence analysis; JGE made the original observation on the presence of an Rpb8 homolog among the "korarchaeal" proteins; all authors read, edited and approved the final version of the manuscript.

\section{Acknowledgements}

EVK and KSM are supported by the Intramural Research Program of the National Institutes of Health, National Library of Medicine. JGE acknowledges the support of korarchaeal genome sequencing by the Joint Genome Institute Community Sequencing Program.

\section{References}

I. Ouhammouch M, Werner F, Weinzierl RO, Geiduschek EP: A fully recombinant system for activator-dependent archaeal transcription. J Biol Chem 2004, 279(50):5I719-5I72I.

2. Werner F, Eloranta JJ, Weinzierl RO: Archaeal RNA polymerase subunits $F$ and $P$ are bona fide homologs of eukaryotic RPB4 and RPB I 2. Nucleic Acids Res 2000, 28(21):4299-4305.

3. Werner F, Weinzierl RO: A recombinant RNA polymerase IIlike enzyme capable of promoter-specific transcription. Mol Cell 2002, 10(3):635-646.

4. Langer D, Hain J, Thuriaux P, Zillig W: Transcription in archaea: similarity to that in eucarya. Proc Natl Acad Sci U S A 1995, 92(13):5768-5772.

5. Zaros C, Briand JF, Boulard Y, Labarre-Mariotte S, Garcia-Lopez MC, Thuriaux P, Navarro F: Functional organization of the Rpb5 subunit shared by the three yeast RNA polymerases. Nucleic Acids Res 2007, 35(2):634-647.

6. Briand JF, Navarro F, Rematier P, Boschiero C, Labarre S, Werner M, Shpakovski GV, Thuriaux P: Partners of Rpb8p, a small subunit shared by yeast RNA polymerases I, II and III. Mol Cell Biol 200 I, 2 I ( I 7):6056-6065.

7. Krapp S, Kelly G, Reischl J, Weinzierl RO, Matthews S: Eukaryotic RNA polymerase subunit RPB8 is a new relative of the OB family. Nat Struct Biol 1998, 5(2): I I0-I I4.

8. Cramer P, Bushnell DA, Kornberg RD: Structural basis of transcription: RNA polymerase II at 2.8 angstrom resolution. Science 200I, 292(5523): I863-1876.

9. Murzin AG: OB(oligonucleotide/oligosaccharide binding)-fold: common structural and functional solution for non-homologous sequences. Embo J 1993, I2(3):86|-867.

10. Barns SM, Delwiche CF, Palmer JD, Pace NR: Perspectives on archaeal diversity, thermophily and monophyly from environmental rRNA sequences. Proc Natl Acad Sci U S A 1996, 93(17):9188-9193.

II. Altschul SF, Madden TL, Schaffer AA, Zhang J, Zhang Z, Miller W, Lipman DJ: Gapped BLAST and PSI-BLAST: a new generation of protein database search programs. Nucleic Acids Res 1997, 25(I7):3389-3402.

12. Lanzendoerfer M, Langer D, Hain J, Klenk HP, Holz I, Arnold-Ammer I, Zillig W: Structure and function of the DNA-dependent RNA polymerase of Sulfolobus . Systematic and Applied Microbiology 1994, 16:656=664.

13. She Q, Singh RK, Confalonieri F, Zivanovic Y, Allard G, Awayez MJ, Chan-Weiher CC, Clausen IG, Curtis BA, De Moors A, Erauso G, Fletcher C, Gordon PM, Heikamp-de Jong I, Jeffries AC, Kozera CJ, Medina N, Peng X, Thi-Ngoc HP, Redder P, Schenk ME, Theriault C, Tolstrup N, Charlebois RL, Doolittle WF, Duguet M, Gaasterland T, Garrett RA, Ragan MA, Sensen CW, Van der Oost J: The complete genome of the crenarchaeon Sulfolobus solfataricus P2. Proc Natl Acad Sci U S A 200I, 98( I 4):7835-7840.

14. Andersson AF, Lundgren M, Eriksson S, Rosenlund M, Bernander R, Nilsson P: Global analysis of mRNA stability in the archaeon Sulfolobus. Genome Biol 2006, 7(10):R99.

15. Pei J, Kim BH, Tang M, Grishin NV: PROMALS web server for accurate multiple protein sequence alignments. Nucleic Acids Res 2007, 35(Web Server issue): W649-52.

16. Edgar RC: MUSCLE: multiple sequence alignment with high accuracy and high throughput. Nucleic Acids Res 2004, 32(5): $1792-1797$

17. McGuffin LJ, Bryson K, Jones DT: The PSIPRED protein structure prediction server. Bioinformatics 2000, 16(4):404-405. 
18. Felsenstein J: Inferring phylogenies from protein sequences by parsimony, distance, and likelihood methods. Methods Enzymol 1996, 266:418-427.

19. Adachi J, Hasegawa M: MOLPHY: Programs for Molecular Phylogenetics. Tokyo, Institute of Statistical Mathematics; 1992.

Publish with Biomed Central and every scientist can read your work free of charge

"BioMed Central will be the most significant development for disseminating the results of biomedical research in our lifetime. " Sir Paul Nurse, Cancer Research UK

Your research papers will be:

- available free of charge to the entire biomedical community

- peer reviewed and published immediately upon acceptance

- cited in PubMed and archived on PubMed Central

- yours - you keep the copyright

Submit your manuscript here:

http://www.biomedcentral.com/info/publishing_adv.asp 